\title{
Analysis on the Development Status of Coal Mine Dust Disaster Prevention Technology in China
}

\author{
Hui Zhang, ${ }^{1}$ Weihai Han, ${ }^{2}$ Youlin Xu ${ }^{1},{ }^{1}$ and Zhenfeng Wang ${ }^{2}$ \\ ${ }^{1}$ Institute of Mining Engineering, Guizhou Institute of Technology, Guiyang 550003, China \\ ${ }^{2}$ School of Energy Science and Engineering, Henan Polytechnic University, Jiaozuo 454003, China \\ Correspondence should be addressed to Youlin Xu; xuyoulin2006@163.com
}

Received 19 February 2021; Revised 29 March 2021; Accepted 8 April 2021; Published 20 April 2021

Academic Editor: Han Wang

Copyright (c) 2021 Hui Zhang et al. This is an open access article distributed under the Creative Commons Attribution License, which permits unrestricted use, distribution, and reproduction in any medium, provided the original work is properly cited.

In recent years, with the increasing level of mechanization, automation, and intelligence in mine mining, dust pollution in the working environment of coal mines has become increasingly serious. Coal mine dust prevention is an important work related to the life, health, and production safety of miners, and it is also one of the technical problems of mine safety in production. With the continuous revision and improvement of China's Occupational Disease Prevention and Control Law, coal mining enterprises have generally strengthened the publicity, prevention, and control of occupational diseases among employees, and the control of coal mine dust has been used as a means for enterprises to improve the production environment and strengthen the occupational health of employees. Key work-based studies have shown that China's coal mines have formed a theoretical system and technology system of dust prevention and control. In the future, China's coal mines will start from intelligent dust prevention, achieve highprecision dust sensing-transmission-assessment and early warning, and develop a combined dust collector that integrates the functions of atomization dust removal, miniaturization, dry and wet mixing, and large air suction capacity. The combined dust collector realizes the efficient ventilation control and dust removal of the fine dust in wide-area complex spaces such as fully mechanized mining face and fully mechanized mining face. At the same time, breakthroughs have been achieved in lowpermeability coal seams, such as strong hydraulic permeability-enhancing technology, intelligent dust-proof robots, and chemical dust suppression. This article introduces the basic concepts, generation, distribution, and hazards of coal mine dust and analyzes the characteristics, applicable conditions, and use effects of various dust control measures such as ventilation dust removal and wet dust removal. Moreover, this article also proposes specific prevention and control measures for related occupational diseases and discusses the development trend of dust prevention and control technology in the hope of providing guidance and reference for coal mine dust prevention and control.

\section{Introduction}

Coal is China's main energy source, accounting for more than $60 \%$ of the primary energy. The status of this subject energy will be difficult to change for a long time in the future. More than $90 \%$ of coal production in China comes from underground mining. Roadways are a necessary passage for coal mines. According to statistics, the total length of roadways newly excavated in coal mines in China each year reaches $12,000 \mathrm{~km}[1,2]$. At present, China's coal mine production safety situation continues to improve and the number of coal mine accidents dropped significantly, but the coal industry has still a relatively high prevalence of pneumoconiosis because, for a very long time, enterprises attached importance to production safety, ignoring occupational health work. As a result, relevant scientific research investment is low, technological progress cannot match the rapid increase in coal production, and the working environment of coal mines cannot be effectively improved; especially, the dust concentration in coal mine workplaces has been too high for a long time and the number of new cases of pneumoconiosis has increased significantly [3]. For nearly 10 years, with the increasing level of intelligence, mechanization, and automation of mines, the probability of accidents and disasters caused by factors such as gas, coal dust, and fire has also increased. In particular, dust pollution in 
coal mines has severely affected the safety of enterprises and threatened the occupational health of workers [4]. According to statistics, the proportion of floating dust generated by various production links in coal mines is as follows: The coal mining face accounts for $50 \%$, the tunneling face accounts for $35 \%$, the shotcrete operation point accounts for $10 \%$, and the coal loading and transportation and unloading links account for 5\% [5], of which the dust production in coal mining, tunneling and bolting, and shotcreting operations accounted for more than $95 \%$ of the mine's total dust production. According to on-site measurements, without any dust prevention measures on the fully mechanized mining face, when the shearer cutting and frameshifting work together, the time-weighted total dust mass concentration in the main work area of personnel can reach $500 \sim 850 \mathrm{mg} / \mathrm{m}^{3}$, even if dust-proof measures are taken, the harsh working environment of the working face cannot be changed [6]. Literature [7] stated that in recent years, anchor spraying operations have mainly adopted dry spraying and wet spraying processes. Although the operation is simple, the free silica content of the dust on the job site is as high as $80 \%$, and the time-weighted dust mass concentration exceeds $200 \mathrm{mg} / \mathrm{m}^{3}$. The above-mentioned dust concentration also greatly exceeds the upper limit set by the state [8]; the number of miners who have pneumoconiosis risk is extremely high. At present, most scholars only conduct research on a single coal mine dust prevention technology, and there are few studies on comprehensive coal mine dust prevention theory and technology systems. Therefore, in order to reflect the latest research results, promote the development of coal mine dust prevention and control technology in China, and improve mine safety and occupational health, this article analyzes the research status of coal mine dust prevention in China, summarizes the achievements of dust prevention theory and technology, and proposes future development directions.

\section{Pneumoconiosis Status of the Coal Industry}

The main occupational hazards in coal mines are dust, high temperature, noise, and toxic and harmful gases. Among them, the proportion of pneumoconiosis caused by dust is the highest. Coal mine pneumoconiosis accounts for more than $70 \%$ of coal mine occupational diseases, and it accounts for more than $55 \%$ of China's pneumoconiosis. Because dust can cause harm to people's health, the "Coal Mine Safety Regulations" stipulates the maximum allowable concentration of total dust and respirable dust in the workplace. The "Regulations on the Prevention of Occupational Hazards in Coal Mine Workplaces" also specifically regulate the concentration of respirable dust in the workplace. Specific criteria are listed in Tables 1 and 2:

Therefore, coal mine dust is the main occupational hazard factor in coal mines. Over the past decade, though China's coal mine production safety situation continues to improve, the coal mine pneumoconiosis reported cases was a higher trend. As can be seen from Figure 1, China coal mine accidents (excluding occupational diseases) in terms of the number of deaths continued to decline since 2005 from more than 6,000 people to 316 people in 2019 . On the contrary, the reported cases of coal mine pneumoconiosis are gradually increasing; especially since 2010, they have been rising sharply, with more than 13,000 cases each year, with an average of more than 14 thousand cases, which is about 2.7 times the average pneumoconiosis embodiment from 2005 to 2009 [9].

In addition to management reasons, the severe status of coal mine pneumoconiosis is also caused by coal mine enterprises and workers' insufficient awareness of the severity of dust hazards and insufficient attention to occupational health. Therefore, the promotion of new technologies is severely constrained, which has led to the coal industry's pneumoconiosis be in serious condition.

\section{The Harm of Coal Mine Dust to Human Health}

The hazards of coal mine dust mainly include four aspects:

(1) The spontaneous combustion nature of coal dust and the explosive nature of coal dust: coal dust explosion hazard in coal mines is one of the most serious disasters. Compared with gas explosions, coal dust explosions have greater intensity and disaster range, more destructiveness, and more serious consequences [10]. The disaster of coal dust explosion in China is very serious. According to statistics, from the beginning of 2000 to the beginning of 2019, 16 coal dust accidents occurred nationwide, resulting in more than 500 deaths.

(2) Coal dust causes pneumoconiosis. A portion of the dust generated in the production process of coal mines is eliminated through measures such as spray dust reduction or ventilation and dust removal, and a portion of the dust particles with smaller particle size is flying and suspended in the production space. For workers, due to the long contact with respirable dust, respirable dust will slowly be deposited in the human lung, for example, small bronchi or alveolar. Prolonged exposure to respiratory dust will produce a series of physiological and pathological changes, leading to lung tissue fibrosis disease [11], called coal worker's pneumoconiosis. In the coal industry, pneumoconiosis is a common occupational disease. Compared with "obvious" coal mine disasters such as gas explosions, pneumoconiosis is more lethal, can damage more groups, and has higher potential harm. According to statistics, the number of deaths due to pneumoconiosis each year is 6 times the number of miners killed in mining disasters and other industrial accidents [12]. According to statistics, since 2010, China has reported an average of 28,000 new cases of occupational diseases each year. As of the end of 2018 , a total of 975,000 cases of occupational diseases have been reported, of which 873,000 cases were occupational pneumoconiosis cases, accounting for about $90 \%$ of the total number of reported cases of occupational diseases. From 2008 to 2018, the total 
TABLE 1: Dust concentration in workplace air standard.

\begin{tabular}{lcc}
\hline The content of free $\mathrm{SiO}_{2}$ in dust $(\%)$ & \multicolumn{2}{c}{ Maximum allowable concentration $\left(\mathrm{mg} / \mathrm{m}^{3}\right)$} \\
Breathable dust
\end{tabular}

TABLE 2: Judgment standard for management limit of dust exposure concentration in coal mine workplace.

\begin{tabular}{lcc}
\hline Type of dust & The content of free $\mathrm{SiO}_{2}$ in dust $(\%)$ & Maximum allowable concentration of respirable dust $\left(\mathrm{mg} / \mathrm{m}^{3}\right)$ \\
\hline Coal dust & $\leq 5$ & 5.0 \\
& $5 \sim 10$ & 2.5 \\
Rock dust & $10 \sim 30$ & 1.0 \\
Cement dust & $30 \sim 50$ & 0.5 \\
\hline
\end{tabular}

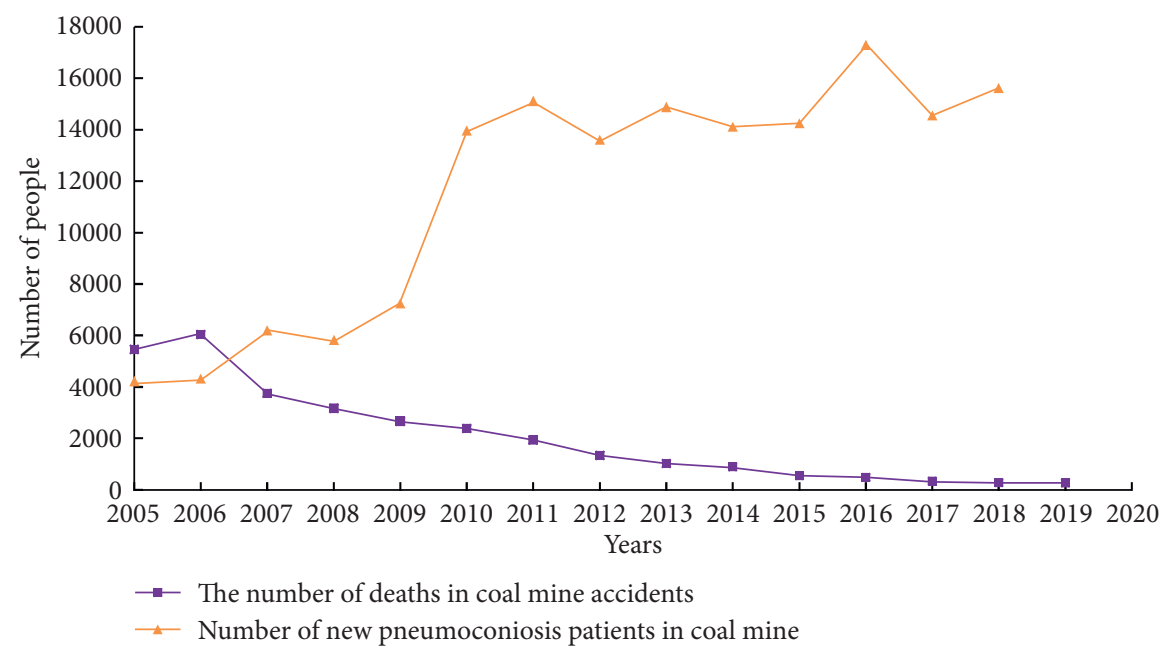

Figure 1: Comparison of the number of new pneumoconiosis and accident deaths in coal mines across the country in recent years.

number of new cases of pneumoconiosis nationwide was 247,611 , of which 125,418 were newly added to coal mine pneumoconiosis, accounting for about $50.65 \%$ [13].

(3) The visibility of the workplace is reduced and workrelated injury accidents are increased.

(4) The wear of the rotating parts of the equipment is accelerated and the working accuracy and life of the equipment are reduced.

\section{The Law of Coal Dust Dispersion Pollution in China}

4.1. Coal Dust Pollution at Fully Mechanized Mining Face. Dust pollution in fully mechanized mining has always been the focus of mine dust prevention and control. The research methods for dust pollution in fully mechanized mining are mainly divided into experiments and numerical simulations. Guo et al. [14] established a mathematical model of dust movement based on the theory of gas-solid two-phase flow. According to the specific properties and measured data of a fully mechanized mining face, the law of dust movement generated by a fully mechanized mining face was clarified. By constructing a two-stage mathematical model, Perret et al. [15] simulated the dust distribution in the mine space and studied the diffusion law of coal dust. Relevant scholars have also used CFD (computational fluid dynamics) numerical calculation methods to study the law of dust migration in fully mechanized mining faces. Seaman et al. [16] used the LES large eddy method to simulate the gas motion process and used the Euler-Lagrange method to numerically simulated the spatial distribution of dust particles. Wang et al. [17] used CFD methods to calculate and simulate the airflow and dust flow characteristics in the mine and, based on the results, proposed two possible dust control schemes (Figures 2 and 3).

Sun et al. [18] used numerical simulation to study the influence of wind flow turbulence on dust pollution and, at the same time, proposed a partial spray closed dust control technology, which improved the dust removal rate (Figure 4). Tan et al. [19] compared the measured dust mass concentration distribution on-site with the simulated coal cutting dust movement law on fully mechanized mining face 


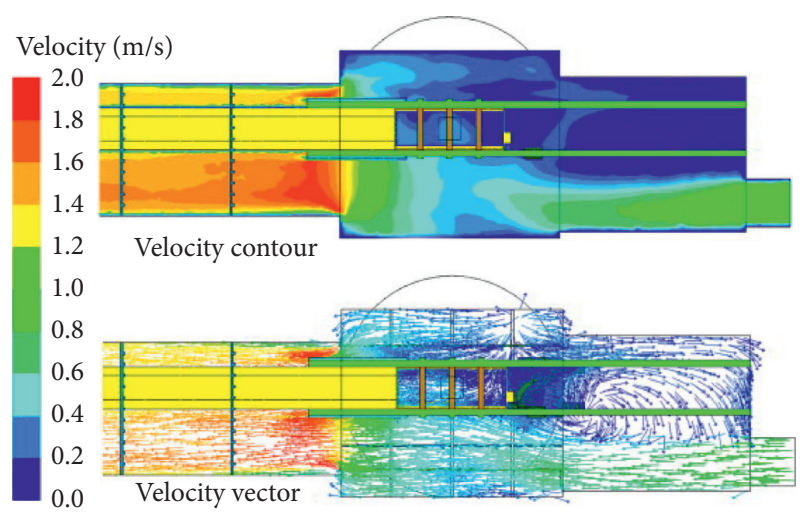

FIGURE 2: Distribution of airflow velocity at the height of breathing zone.

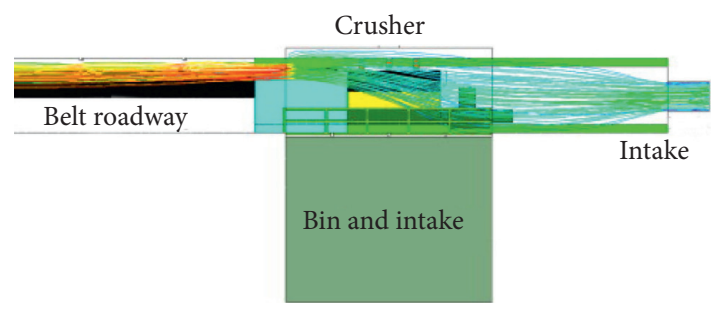

FIgURE 3: Inlet air streamline diagram.

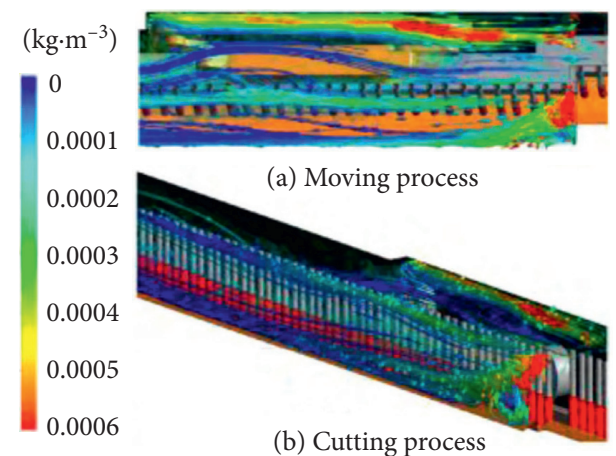

FIGURE 4: Dust concentration movement trace diagram.

and obtained several important factors that affect the dust quality concentration on fully mechanized mining face, mainly the wind speed of the working face, the speed of the shearer drum, the speed of the scraper conveyor chain, and the coal wall surface conditions. Yao et al. [20] conducted numerical simulations on the airflow movement and coal dust distribution law of fully mechanized mining face under different ventilation conditions and obtained the dust reduction mechanism of upward ventilation and downward ventilation and the optimal dust exhaust wind speed of large inclination fully mechanized mining face.

In summary, domestic and foreign scholars have conducted research on the law of dust pollution on fully mechanized coal mining faces through experiments and numerical simulations. According to the characteristics of different dust sources on a fully mechanized mining face, a mathematical model of airflow-dust particle DPM coupled flow suitable for a fully mechanized mining face is established, and the Euler-Lagrange method is used to describe the turbulent diffusion of airborne dust. The CFD numerical simulation of the dispersion process of the coal dust generated by the shearer cutting on the fully mechanized working face, the coal dust generated by the moving frame, and the coal dust carried by the ventilation is carried out. The results obtained have laid a scientific theoretical foundation for dust prevention and control in a fully mechanized mining face.

\subsection{Coal Dust Pollution at Comprehensive Excavation Face.} There are many factors affecting the dust dispersion in the comprehensive mechanized tunneling face, such as the changeable position of dust production, and the complicated technical process, and the researchers have conducted indepth and detailed research. Wen et al. [21] used a combination of experiment and numerical simulation to study the movement of wind and dust on the fully mechanized excavation face. The research results show that there is a corresponding relationship between the airflow streamline and the dust trace, and the test and simulation results are consistent with the field measurement. Wu et al. [22] analyzed the dust generation mechanism of the fully mechanized excavation face and used the Fluent software to conduct a numerical simulation study on the dust distribution and migration law of the working flour to obtain the overall dust migration and distribution law.

\section{The Theory of Coal Dust Wetting}

Coal seam water injection and spray dust reduction are the main measures to prevent and control dust on the mining face, water injection into the coal seam to be mined through boreholes, or fog field generated by high-pressure atomization and can moisten the coal (dust), thereby reducing the floating coal produced during the mining process. Therefore, the wettability of pulverized coal is an important factor affecting the dust reduction effect. For this reason, in order to better understand the wettability of coal dust, it is first necessary to analyze and study the solid-liquid relationship between coal dust and water. In this regard, after a detailed analysis of the surface wettability of ultrafine coal powder, Abhishek Kumar et al. [23] found that with the degree of deterioration, the surface hydrophobicity of ultrafine coal powder after pulverization will continue to increase. Zhao et al. [24] analyzed the characteristics of coal surface free energy and the microscopic mechanism of coal adsorption of water based on the structural characteristics of coal macromolecules and surface and concluded that coal adsorption of water molecules is multilayer adsorption. The first layer of water absorption is mainly due to the hydrogen bonding between coal and water molecules, while the adsorption of other water molecular layers is caused by long-range forces between molecules. Sergei et al. [25] analyzed the movement process of water in the coal body when water was injected into the coal seam, applied the theory of interface chemistry to analyze the wetting process of water on the coal surface, 
summarized the conditions under which the coal body can wet by itself, and the wetting mechanism of water on the coal surface is preliminarily explained.

Kollipara et al. [26] studied their physical properties, mineralogical properties, and wetting properties for different dust samples. The fixed time wettability (trying to simulate the wettability around the mining environment) and absolute time wettability (assessing internal wetting rate) are used to evaluate the wettability of dust, and it is concluded that the fixed wettability of coal dust is $57 \%$ to $99 \%$, the wettability of most mines is above $90 \%$, and the wettability in the middle of the coal seam is the worst. The contact time between dust particles and water droplets is an important factor in improving the wettability of coal dust. The results show that it takes more time for dust with larger particles to be completely wetted. Arkhipov et al. [27] proposed a new method for estimating the wettability of fine coal particles on water droplets, which improved the accuracy of particle wettability estimation. The combination of wet dust removal and chemical dust suppression, especially anionic surfactants, has the most significant impact on coal wettability. Li et al. [28] and others systematically analyzed the physical properties of coal dust and its wetting behavior. The effects of different surfactants on the wettability of coal dust were studied and compared with deionized water. The research results show that the finer the particle size of the coal, the more complex the microstructure of the coal, and the worse the wettability of the coal. Among the three different coal dust samples, the coal with a higher volatile content has poorer wetting performance because the volatiles are easier to release and a gas film is more likely to form around the particles.

\section{Theory of Dust Mist Condensation and Wet Dust Removal}

Due to the particularity of coal mine production conditions, in addition to coal seam water injection prewetting coal body technology, dust mist-condensation and wet dust suppression technology is currently an effective measure generally adopted in coal mining face in China. Zhou and Wang [29] believed that the most obvious factor that affects the trapping of dust particles by fog droplets is the droplet diameter. The small diameter of the droplets makes it easier to capture dust particles. However, during the actual dust reduction operation, if the diameter of the formed droplets is too small, it will cause the droplets to evaporate too quickly and the retention time is too short, which will affect the overall dust collection efficiency. Lin et al. [30] studied the gas-liquid ratio of single-phase nozzles and two-phase nozzles, as well as the variation law between axial distance and radial distance. Zhou et al. [31] combined experiments and theoretical analysis to study the spray parameters of the dust suppression system and obtained the relationship between pressure and other parameters. In order to correctly evaluate the interaction between water droplets, dust, and flowing airflow, Swansona and Langefeld [32] established a wind tunnel to simulate underground operations under different environmental conditions, evaluated the control conditions and requirements of each dust source, and developed an optimized dust source control and dust removal system. Tessum and Raynor [33] used a pneumatic particle size analyzer to measure the particle size and concentration of the charge-separated particles and studied the dust collection performance of different types of surfactants on different coal dust particle sizes and charges. The above studies have promoted the development of dust-mist-condensation and wet-type dust reduction technology. However, the current spray dust reduction measures in fully mechanized mining face still have problems such as the lack of scientificity in nozzle selection and layout and poor spray dust reduction effects. In order to further improve the efficiency of spray dust reduction, the current more advanced theories are mainly to improve the dust mist-condensation mechanism from a microscopic point of view by measuring the droplet size, velocity, and concentration distribution of different types of nozzles [34], formed a system of nozzle atomization characteristics and microspray dust reduction mechanism, and developed a new type of high-efficiency single-water and Fengshui atomizing nozzles, which can be made of copper, stainless steel, ceramics and other forms (Figure 5).

\section{Ventilation and Dust Removal in China}

Ventilation and dust removal are to use the mine ventilation system to dilute and discharge the dust in the mine air to prevent excessive dust accumulation and concentration exceeding the limit. Doing a good job of ventilation is an important part of achieving a good dust-proof effect. Ventilation and dust removal methods and measures for tunneling working face are as follows:

(1) The most commonly used method is to use the local ventilator ventilation method. This kind of ventilation method has three types: press-in type, extraction type, and mixed type, and mixed type is generally used.

(2) Technology is used to control the dust-laden airflow to diffuse outwards, such as wall air ducts and highpressure wind shielding.

(3) The long-pressure short-extraction dust removal system is matched with the roadheader and dust collector to comprehensively reduce dust.

(4) Take wind-guiding measures, set up wind barriers, or use airflow sprayers to guide the wind to isolate dust.

Ventilation and dust removal methods and measures for fully mechanized mining faces are as follows:

(1) Select the best ventilation parameters (the best dust exhaust wind speed is $1.5 \sim 4 \mathrm{~m} / \mathrm{s}$ ) to ensure the effect of ventilation and dust removal.

(2) Change the ventilation system or the direction of airflow at the working face (W-type and E-type ventilation systems are the best, and adopting the direction of coal flow; that is, downward ventilation can greatly reduce the concentration of working dust). 


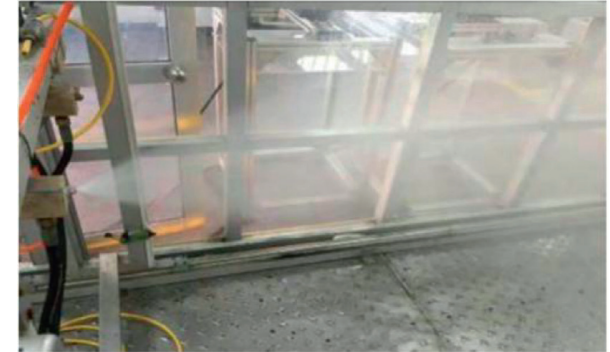

Figure 5: Atomization effect of $4 \mathrm{MPa}$ pressure nozzle.

(3) Install simple ventilation facilities to reduce air leakage and ensure air supply. The main methods include setting goaf air curtains, sidewalk air curtains, and coal shearer dust curtains.

\section{Wet Dust Removal in China}

8.1. Wet Rock Drilling. Wet rock drilling technology is that when a rock drill (or electric coal drill, bolter, drill truck, etc.) is drilling, it sends pressure water to the bottom of the borehole to moisturize, flush, and discharge the dust generated by the rotation of the drill bit. This technology can effectively reduce the amount of dust produced, and the dust reduction rate can reach about $90 \%$.

8.2. Dust Reduction by Spray. Dust reduction by spray is the spraying of water stream into fine water droplets under the rotation and impact of sprayer (also called nozzle). At present, the commonly used spray dust reduction technologies are as follows:

(1) The mining unit sprays mainly include internal and external spray dust reduction, radial fog screen dust reduction, high-pressure spray negative pressure dust reduction, and dust-containing airflow control dust reduction.

(2) Interrack spraying includes nozzles installed at the front beam of the support, the front and back of the racks, and the coal caving port to achieve simultaneous spraying and dust reduction when raising, lowering, and moving the rack.

8.3. Sprinkle Water to Reduce Dust. Sprinkle water to reduce dust is to wet the deposited coal dust with water so that the coal dust can be condensed into larger particles that can adhere to the surface of the coal and rock, ensuring that it is not easy to fly during shipment. General sprinkling has a poor dust reduction (low pressure) effect and large water consumption. At present, a new technology of high-pressure sprinkling (water pressure greater than $910 \mathrm{kPa}$ ) has emerged, which makes sprinkling and dust reduction measures more perfect.

8.4. Water Cannon Mud. Water cannon mud is a plastic bag filled with water to replace part of the cannon mud in the blasthole. During blasting, water vaporization and dust condensation achieve the dust reduction effect, which can reduce the dust concentration by $20 \%$ to $50 \%$.
8.5. Coal Seam Water Injection. Coal seam water injection is to inject pressurized water into the borehole under the action of the water injection system to prewet the native coal dust in the coal body and at the same time make the entire coal body surrounded by water, thereby playing the role of dust reduction and dust suppression. Coal seam water injection is active and effective in reducing dust and has a continuous dust prevention effect. At present, long-hole water injection is widely used internationally.

\section{Purifying Airflow}

Purifying airflow is to install dust-catching facilities in the working face or roadway to build a dust barrier to purify the dust-laden air (airflow). At present, purified water curtain, catching dust net, and wet dust removal device are commonly used.

(1) Purified water curtain: the full-face water curtain formed by the water flow from multiple nozzles installed on the top of the roadway and the working face has the effect of purifying the inlet airflow and reducing the dust concentration of the return airflow. At present, the purified water curtain has specific forms such as microhole spray, nozzle spray, fine water spray, and Feng Shui linkage spray. There are various control methods such as manual, photoelectric, touch, and mechanical transmission. The dust reduction rate of the water curtain can reach $70 \%$ to $95 \%$.

(2) Catching dust net: install a net in the roadway that can pass airflow and collect dust. It is generally used in conjunction with water curtains and sprays.

(3) Wet dust removal device: the device removes dust by a collision between dust particles and liquid droplets. At present, China has wet dust collectors such as SCF series dust collectors, KGC series tunneling machine dust collectors, TC series tunneling machine dust collectors, and MAD series airflow purifiers.

\section{Airtight Dust Extraction}

Airtight dust extraction is to use a dust cover to close the local dust production point, and draw the dust-containing air through the pipeline to the dust collector or dust collector to filter, collect, purify and discharge. It is suitable for the situation where water shortage or water cannot be used and the dust source needs to be sealed, common dust-catching aperture, and hole bottom catching dust in two ways. This kind of dry dust-catching method has no water mist in the workplace, the air is dry, and the compressed air consumption is large.

\section{Personal Protection}

Personal protection refers to wearing dust-proof equipment to filter and purify dusty air, so that workers can breathe clean air. Individual dust-proof appliances mainly include dust-proof caps, dust-proof masks, and dust-proof 
respirators. With the advancement of science and technology, advanced protective equipment, such as self-priming filter dust masks, power supply air filter dust masks (air supply masks and airflow helmets), and compressed air respirators, has been developed.

\section{Prevention and Control Measures of Coal Mine Dust and Related Occupational Diseases}

Through the analysis of the causes of coal mine dust-related occupational diseases, long-term, large-scale exposure and inhalation of fine dust particles are the root causes of the disease. For coal mine dust control and related occupational disease prevention, measures should be taken from the following aspects:

(1) Improve the awareness of occupational disease prevention and control of enterprise managers and increase relevant investment. First of all, coal mine managers should raise their awareness of occupational disease prevention and control, clarify the main responsibility of enterprises in the prevention and control of occupational diseases, and establish a people-oriented concept. Based on the principle of observing regulations and caring for employees, we earnestly implement the "Occupational Disease Prevention and Control Law" and take concrete and effective measures to reduce the generation of mine dust and reduce the harm to personnel. In terms of organization and management, a special mine dust prevention management department should be established, equipped with full-time dust prevention technical personnel, and implement the "three simultaneous" occupational disease protection facilities. Increase investment in mine dust prevention and control, set up special funds, timely update mine dust removal equipment, purchase dust masks, face masks, and other labor protection products for employees, and supervise employees to wear and use them correctly.

(2) Improve the production process to reduce dust generation. For coal shearers, roadheaders, crushers, loading and unloading machinery, self-moving hydraulic supports, and other equipment, an automatic spray device and a dust cover can be installed, and a magnetized water device can be added to enhance the water mist absorption and dust reduction capabilities. In the process of rock tunnel excavation, wet drilling and wet rock drilling should be promoted on the blasting working surface, and the dust in the blasthole should be moistened and washed with pressure water. At the same time, automatic water curtain purification measures should be adopted to achieve the purpose of dust reduction. When the rock formation conditions are not suitable for the wet drilling method, the dry drilling method can be used with a dry dust catcher to catch dust.
(3) Improve mine ventilation and purify air flow. The "Coal Mine Safety Regulations" stipulate that the minimum wind speed during the excavation of rock tunnels shall not be less than $0.15 \mathrm{~m} / \mathrm{s}$. At this wind speed, the mineral dust below 5 microns can be suspended and mixed evenly with the air and discharged with the wind flow. In order to better dilute and discharge the coal dust, the wind speed can be appropriately increased in the operation sites with large dust production and high coal dust concentration, but if the wind speed is too high, the dust content in the air will increase. Therefore, the "Coal Mine Safety Regulations" stipulates that the maximum allowable wind speed at the coal mining face is $4 \mathrm{~m} / \mathrm{s}$.

(4) Strengthen education and training to improve employees' awareness of occupational disease prevention. Most coal mine enterprises lack the awareness of self-protection due to the poor overall quality of their employees and lack of awareness of the dangers of coal mine dust. They often do not use labor protection equipment and dust-proof facilities as required at work. In this regard, the education and training of coal mine employees should be strengthened to make them fully aware of the hazards of coal mine dust to human health so as to increase their awareness of occupational disease prevention and actively use dust removal equipment as required.

\section{Prospects of Research on Coal Mine Dust Prevention in Cina}

In recent years, with the increasing level of mechanization, automation, and intelligent mining in China's coal mines, dust pollution in the operating environment has become more and more serious, which has greatly affected mine safety production and seriously threatened the occupational health of miners. Scholars at home and abroad have actively explored mine dust prevention and control and have achieved many innovative results. However, different mining areas in China's coal mines have great differences in coal seam conditions, mining methods, technical equipment levels, and comprehensive management levels. There is still a lot of room for improvement in the development of coal mine dust prevention theory and technology. Based on this, in the next period of time, the focus of coal mine dust prevention research will be mainly in the following aspects:

(1) Develop high-precision, wide measuring range of respirable dust sensor to achieve continuous online monitoring of the mass concentration of respirable dust and to the direction of miniaturization, longdistance transmission, large-area coverage, and continuous monitoring.

(2) Through the research on the "Set of Coal Mine Dust Monitoring Technology," the mass concentration, toxicity, and other parameters of respirable dust are collected and combined with the accumulated dust 
exposure time, dust physicochemical characteristics, and other indicators, the existing research results of pneumoconiosis are used to establish an early warning indicator system and discrimination model for pneumoconiosis. On this basis, according to the pneumoconiosis danger circle theory and the pneumoconiosis excess risk classification method, prepare to establish a coal mine dust hazard warning system to realize dust hazard warning, and promote the optimization of dust prevention and control measures, scientifically guide the monitoring and management of dust prevention and pneumoconiosis.

(3) Carry out research on high-pressure hydraulic antireflection technology for low-permeability, hydrophobic, and difficult-to-inject coal seams, artificially create cracks or fissures in the coal seam in advance, and by adding a wetting agent, improve the wettability of the hydrophobic hard-to-water-injection coal seam, thereby improving the water injection effect of the hard-water-injection coal seam. Study the coupling technology of gas drainage and coal seam water injection so as to effectively suppress the dust intensity during coal seam mining.

(4) Study the key technologies and equipment for the treatment of respirable dust hazards in coal mines and effectively reduce the mass concentration of respirable dust. Research and promote the application of monorail hoisting technology in fully mechanized excavation face to realize the synchronous movement of dust removal system and roadheader. Study the ventilation and dust removal system for rock tunnel excavation.

(5) Research and development of coal mine dust monitoring equipment remote online monitoring system, improve the management capacity of the downhole equipment.

\section{Conclusions}

(1) Through the research on the "Set of Coal Mine Dust Monitoring Technology," the mass concentration, toxicity, and other parameters of respirable dust are collected and combined with the accumulated dust exposure time, dust physicochemical characteristics, and other indicators, the existing research results of pneumoconiosis are used to establish an early warning indicator system and discrimination model for pneumoconiosis. On this basis, according to the pneumoconiosis danger circle theory and the pneumoconiosis excess risk classification method, prepare to establish a coal mine dust hazard warning system to realize dust hazard warning, and promote the optimization of dust prevention and control measures, scientifically guide the monitoring and management of dust prevention and pneumoconiosis.
(2) Carry out research on high-pressure hydraulic antireflection technology for low-permeability, hydrophobic, and difficult-to-inject coal seams, artificially create cracks or fissures in the coal seam in advance, and by adding a wetting agent, improve the wettability of the hydrophobic hard-to-water-injection coal seam, thereby improving the water injection effect of the hard-water-injection coal seam. Study the coupling technology of gas drainage and coal seam water injection so as to effectively suppress the dust intensity during coal seam mining.

(3) Study the key technologies and equipment for the treatment of respirable dust hazards in coal mines and effectively reduce the mass concentration of respirable dust. Research and promote the application of monorail hoisting technology in fully mechanized excavation face to realize the synchronous movement of dust removal system and roadheader. Study the ventilation and dust removal system for rock tunnel excavation.

(4) Research and development of coal mine dust monitoring equipment remote online monitoring system improve the management capacity of the downhole equipment.

\section{Data Availability}

The data used to support the findings of this study are available from the corresponding author upon request.

\section{Conflicts of Interest}

The authors declare that they have no conflicts of interest.

\section{Acknowledgments}

This work was supported by the National Natural Science Foundation of China (51764010 and 51874109) and the Science and Technology Project for Outing and Young Talents of Guizhou (Talents of Science Platform in Guizhou [2019] 5674).

\section{References}

[1] H. Kang, "Support technologies for deep and complex roadways in underground coal mines: a review," International Journal of Coal Science \& Technology, vol. 1, no. 3, pp. 261-277, 2014.

[2] H. Kang, "Sixty years development and prospects of rock bolting technology for underground coal mine roadways in China," Journal of China University of Mining and Technology, vol. 45, no. 6, pp. 1071-1081, 2016.

[3] F. Wu, J. Chen, and J. Wang, "Development of dust sampler based flow closed-loop control," Acta Metrologica Sinica, vol. 38, no. 1, pp. 87-90, 2017.

[4] K. J. Candra, S. A. Pulung, and M. A. Sadashiv, "Dust dispersion and management in underground mining faces," International Journal of Mining Science and Technology, vol. 24, no. 1, pp. 39-44, 2014.

[5] R. Tong, M. Cheng, X. Yang, Y. Yang, and M. Shi, "Exposure levels and health damage assessment of dust in a coal mine of 
Shanxi Province, China," Process Safety and Environmental Protection, vol. 128, pp. 184-192, 2019.

[6] S. Li, B. Xie, S. Hu et al., "Removal of dust produced in the roadway of coal mine using a mining dust filtration system," Advanced Powder Technology, vol. 30, no. 5, pp. 911-919, 2019.

[7] G. Zhou, W. Yin, and B. Feng, "Numerical simulation on the distribution characteristics of dust-droplet field during support movement in a fully-mechanized mining face and related engineering applications," Journal of China Coal Society, vol. 43, no. 12, pp. 3425-3435, 2018.

[8] Y. Niu, L. Zhang, and B. Shi, "Experimental study on the explosion-propagation law of coal dust with different moisture contents induced by methane explosion," Powder Technology, vol. 361, pp. 507-511, 2020.

[9] M. M. Biliaiev, V. V. Biliaieva, V. A. Kozachyna, O. V. Berlov, M. O. Oladipo, and P. S. Kirichenko, "Reducing of coal dust release from train wagon with barrier," IOP Conference Series: Materials Science and Engineering, vol. 985, p. 12018, 2020.

[10] M. Tousif, A. Harish, S. Muthu Kumaran, and V. Raghavan, "Numerical study of interaction of coal dust with premixed fuel-lean methane-air flames," Advanced Powder Technology, vol. 31, no. 9, pp. 3833-3844, 2020.

[11] L. Mao, L. Peng, and H. Wang, "Consensus of Chinese experts on pneumoconiosis treatment," Environment and Occupational Medicine, vol. 35, no. 8, pp. 677-689, 2018.

[12] Y. Lin, Y. Ge, W. Shi et al., "Review of current research on occupational disease prevention in China," Chinese Health Law, vol. 3, no. 4, pp. 20-22, 2010.

[13] L. Dong, X. Tong, X. Li, J. Zhou, S. Wang, and B. Liu, "Some developments and new insights of environmental problems and deep mining strategy for cleaner production in mines," Journal of Cleaner Production, vol. 210, no. 10, pp. 1562-1578, 2019.

[14] Q. Guo, W. Ren, and J. Shi, "Foam for coal dust suppression during underground coal mine tunneling," Tunnelling and Underground Space Technology, vol. 89, pp. 170-178, 2019.

[15] J. L. Perret, S. Miles, F. Brims et al., "Respiratory surveillance for coal mine dust and artificial stone exposed workers in Australia and New Zealand: a position statement from the Thoracic Society of Australia and New Zealand," Respirology, vol. 25, no. 11, pp. 1193-1202, 2020.

[16] C. E. Seaman, M. R. Shahan, T. W. Beck, and S. E. Mischler, "Design of a water curtain to reduce accumulations of float coal dust in longwall returns," International Journal of Mining Science and Technology, vol. 30, no. 4, pp. 443-447, 2020.

[17] J. Wang, W. Zhao, J. Zhao, B. Liu, and L. Song, "Effect of water quality on wettability of coking coal dust," IOP Conference Series: Earth and Environmental Science, vol. 545, p. 12032, 2020.

[18] B. Sun, W. Cheng, J. Wang, and H. Wang, "Effects of turbulent airflow from coal cutting on pollution characteristics of coal dust in fully-mechanized mining face: a case study," Journal of Cleaner Production, vol. 201, pp. 308-324, 2018.

[19] C. Tan, Z. Jiang, J. Chen et al., "Numerical simulation of influencing factors of dust migration in fully mechanized coal mining," Journal of University of Science and Technology Beijing, vol. 36, no. 6, pp. 716-721, 2014.

[20] X. Yao, G. Lu, K. Xu et al., "Ventilation and dust reduction system of large angle fully mechanized caving face based on fluent," Journal of Northeast University: Natural Science Edition, vol. 35, no. 10, pp. 1497-1501, 2014.

[21] N. Wen, W. Wei, M. Xiao, Y. Liu, H. Peng, and Q. Liu, “The effects of ventilation parameters on the migration behaviors of head- on dusts in the heading face," Tunnelling and Underground Space Technology, vol. 70, pp. 400-408, 2017.

[22] M. Wu, H. Xiangming, Z. Qian, L. Wei, Z. Yanyun, and H. Zhenglong, "Study on preparation and properties of environmentally-friendly dust suppressant with semi-interpenetrating network structure," Journal of Cleaner Production, vol. 259, p. 120870, 2020.

[23] T. Abhishek Kumar, S. Prasad, and S. Ray, "Laboratory investigation on the impact of coal dust deposition on PVPanel performance," IOP Conference Series: Materials Science and Engineering, vol. 993, p. 12088, 2020.

[24] Q. Zhao, J. Liu, C. Huang et al., "Characteristics of coal dust deflagration under the atmosphere of methane and their inhibition by coal ash," Fuel, vol. 291, pp. 120-121, 2021.

[25] A. Sergei and O. Konstantin, "Mathematical modeling of coal dust screening by means of sieve analysis and coal dust combustion based on new methods of piece-linear function approximation," Applied Sciences, vol. 11, no. 4, p. 1609, 2021.

[26] V. K. Kollipara, Y. P. Chugh, and K. Mondal, "Physical, mineralogical and wetting characteristics of dusts from interior basin coal mines," International Journal of Coal Geology, vol. 127, pp. 75-87, 2014.

[27] V. A. Arkhipov, D. Y. Paleev, Y. F. Patrakov, and A. S. Usanina, "Coal dust wettability estimation," Journal of Mining Science, vol. 50, no. 3, pp. 587-594, 2014.

[28] Q. Li, B. Lin, S. Zhao, and H. Dai, "Surface physical properties and its effects on the wetting behaviors of respirable coal mine dust," Powder Technology, vol. 233, pp. 137-145, 2013.

[29] J. Zhou and H. Wang, "Improvement and optimization of spray dust control system for fully mechanized coal face shearer," Mechanical and Electrical Engineering, vol. 32, no. 2, pp. 211-214, 2015.

[30] Y. Lin, Y. Ge, W. Shi et al., "Experimental study and numerical simulation on spray characteristics of different nozzle," Chinese Health Law, vol. 35, no. 12, pp. 20-22, 2010.

[31] G. Zhou, N. Wen, W. Cheng et al., "Influence regulations analysis of high-pressure atomization dust-settling to dust particle's microscopic parameters in fully mechanized caving coal face," Journal of China Coal Society, vol. 39, no. 10, pp. 2053-2059, 2014.

[32] L. Swanson and O. Langefeld, "Fundamental research in water spray systems for dust control," Mining Technology, vol. 124, no. 2, pp. 78-82, 2015.

[33] M. W. Tessum and P. C. Raynor, "Effects of spray surfactant and particle charge on respirable coal dust capture," Safety and Health at Work, vol. 8, no. 3, 2017.

[34] G. Zhou, W. Cheng, G. Wang, and X.-F. Cui, "Experiment research of the coupling relationship between dust field and droplet field about fully mechanized and roof caving workface," Journal of China Coal Society, vol. 35, no. 10, pp. 1660-1664, 2010. 